\title{
A STUDY OF THREE CASES OF TUMOR OF THE BRAIN, IN WHICH OPERATION WAS PERFORMED - ONE RECOVERY, TIVO DEATHS. ${ }^{\prime}$
}

BY LEO. STIEgl,TTZ, M.D., NEW YORK.

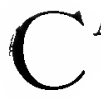

ASE I. Glio-sarcoma. The size of an almond; developing two years and ten months after the successful operation of a cystic tumor of the left motor area. Successful localization and excision. Recovery.

The symptoms in this case prior to the first operation in June, I 892, consisted of focal epileptic discharges in the right arm with occasional general convulsions. Three months after the onset of the focal epileptic dis. charges, paralysis began to develop in the muscles affected by the focal seizures. In spite of the absence of all general cerebral symptoms, notably headache and choked disc, the diagnosis was made of a tumor of the brain, involving part of the arm centre, and an operation was recommended. The operation was performed by $\mathrm{Dr}$. Arpad G. Gerster in June, I892, and revealed the pre. sence of a small cystic tumor, which was evacuated and allowed to heal under drainage. The walls of the cyst were perfectly smooth, and no sign of any further growth was found. The patient made a good recovery. Excepting occasional focal discharges and three or four general convulsions, she remained perfectly well for the next two and a half years. In February, I895, she complained for the first time of headache, which was quite severe and persisted for two weeks. At the same time the signs of a beginning papillitis were found, and the right arm, which had recovered a good part of its original power, showed again a marked loss of power. The diagnosis was made of a refilling of the old cyst or a recurrence of the new growth upon the basis of which the cyst had probably originally developed, and ele-

' Read by title at the Meeting of the American Neurological Association, held in Boston, June 8, r895. 
ments of which had in all probability been left in the wall of the cyst. Another operation being urged, Dr. Gerster entered the skull at the old aperture, which had been left covered by a gold plate. A small new growth, not larger than an almond, was exposed at the point where the old cyst had been. This new growth was carefully excised by Dr. Gerster, and then a circle of brain tissue which had surrounded it, and which was apparently partly infiltrated by the neoplasm was excised. The patient again made a good recovery, excepting three seizures which occurred immediately upon the removal of the original dressing. The patient has been well since the operation; the paralyzed arm is regaining its power slowly. The microscopic examination of the new growth revealed the presence of the old cyst wall, and starting from one point of it a glio-sarcoma; the surrounding brain tissue, which had been excised, was found partly infiltrated with elements of a glio melano. fibro-sarcoma.

The author draws the following conclusions from the case:

I. A new growth may be present in the brain without causing any general cerebral symptoms whatsoever; in order to make an early diagnosis of the presence of a new growth it is well, therefore, to be guided by the symptoms present rather than by the absence of other customary symptoms.

2. A cystic tumor of the brain not of parasitic origin is not as innocent in character as many would believe.

3. The simple evacuation of such a cyst when found, is not sufficient; if it is surgically possible, the wall and the surrounding brain tissue should be excised in order to prevent a future growth of the malignant elements left in the wall of the evacuated cyst.

4. If the immediate excision is not possible, it would be advisable to open the wound a few months later and attempt to excise the collapsed cyst wall, which is com. pressed into a small space by the general cerebral pressure in the same way in which an abcess cavity is quickly obliterated after evacuation.

CASE II. Glio sarcoma of the right cerebellar hemisphere--successful localization, operation, death, autopsy.

The symptoms offered by the patient during life were headache, choked discs, vomiting, spells of vertigo, occasional staggering to the right side, deafness in 
the right ear, partial reaction of degeneration to the electric currents in the right side of the face, although no trace of paralysis was present till some months later, and hyperæsthesia to the galvanic current, with reversal of formula in the left ear. The diagnosis was made of a new growth of the cerebellum compressing the right seventh and eighth nerves, prior to, or at, their common entrance at the internal auditory meatus. The patient's condition became so miserable that it was agreed to make an effort to relieve him by surgical means. Dr. Gerster proposed entering the skull in the region of the right occipital lobe, raising the latter and entering the posterior cranial fossa by an opening through the tentorium. On October 27, I893, the operation was performed; there was tremendous intra. cranial pressure, producing quickly a large untractable hernia cerebri; although Dr. Gerster succeeded in exposing the tentorium, the operation could not befcompleted on account of the great intra-cranial pressure. The patient rallied well from the operation itself; the wound was infected during a change of dressings, how. ever, and the patient died of purulent meningitis. At the autopsy a glio-sarcoma of the cerebellum, of the size of a large walnut, was found at the point to which the symptoms during life had pointed.

CASE III. Spindle-cell sarcoma of the left arm, face and speech centres-successful localization, operation, death, autopsy.

The symptoms presented were the occurence of five or six focal epileptic seizures, involving the right side of the face and the right arm, slight loss of power in the right face and arm, motor aphasia, headache, mental hebetude, and a point in the left fronto-parietal region of the skull which was exquisitely sensitive to tapping. The diagnosis was made of a brain tumor, involving the face and probably the arm and speech centres in the lower half of the left central convolution; on account of the in. volvement of the motor speech centre rather forward than backward of the fissure of Rolando. After repeated aelays the operation, which had been recommended as soon as specific treatment had proved futile, was performed by Dr. Howard Lilienthal. By the time the dura was well exposed the cachetic patient had lost so much blood, the diploe bleeding profusely, that it was decided to postpone opening the dura to a subsequent operation. The patient, however, never rallied from 
the shock of the operation and the loss of blood, and died twenty-nine hours after the operation. The autopsy showed the presence of a sarcoma involving the arm, face and motor specch centres in the left motor area, the opening in the skull corresponded to the upper half of the tumor; had the dura been opened, the new growth would have been exposed; the growth was imbedded in the brain substance in such a way that it could have been enucleated and removed through an opening sufficiently large. 